\title{
SUR LA SPATIALITÉ DE L’ÉCRITURE - LE PAYSAGE ET LA CARTE
}

\author{
Ramona PÁL-KovÁCS \\ Université de Szeged, Hongrie
}

\begin{abstract}
En): After a brief summary of the spatial turn, which consists of the reinsertion of space and place into social sciences and humanities, this paper outlines the possibilities of a spatial reading of literature. For this, two main concepts are examined, landscape and map, in order to be able to outline a theoretical framework in which a spatial analysis of literary texts proves to be fruitful.
\end{abstract}

Keywords (En): spatial turn ; landscape ; map ; mapping ; literature ; Michel Collot ; Michel Foucault ; Gilles Deleuze ; Denis Cosgrove ; Robert Tally ; Bertrand Westphal ; geocriticism ; geopoetics ; literary cartography ; literary geography

Mots-clés (Fr) : tournant spatial ; paysage ; carte ; cartographie ; littérature ; Michel Collot ; Michel Foucault; Gilles Deleuze; Denis Cosgrove; Robert Tally; Bertrand Westphal; géocritique ; géopoétique ; cartographie littéraire ; géographie littéraire

Au début des années 1980, on assiste au «tournant spatial » ou «tournant géographique » des sciences sociales et humaines. Ce changement de perception est visible également dans les études littéraires. Gilles Deleuze et Félix Guattari introduisent leur géophilosophie par l'hypothèse que la pensée «n'est ni un fil tendu entre un sujet et un objet, ni une révolution de l'un autour de l'autre. Penser se fait plutôt dans le rapport du territoire et de la terre » (DELEUZE - GUATTARI, 1991 : 82). Ce tournant de la pensée est le résultat d'un déplacement progressif de l'intérêt pour la temporalité vers la spatialité, qui caractérise notre époque. Comme Michel Foucault le constate dans Des espaces autres en 1967, notre époque est «l'époque de l'espace [...] l'époque du simultané, [...] l'époque de la juxtaposition, [...] l'époque du proche et du lointain, du côte à côte, du dispersé » (FOUCAULT, 1994 : 752). Contrairement au $19^{\mathrm{e}}$ siècle obsédé par l'histoire, le $20^{\mathrm{e}}$ siècle se tourne vers l'espace. Cela ne signifie pas que cet espace est dépourvu de temporalité, il s'agit plutôt d' "une certaine manière de traiter ce qu'on appelle le temps et ce qu'on appelle l'histoire » (FOUCAULT, 1994 : 752). Foucault introduit dans son article le concept d'hétérotopie pour désigner un certain espace ayant «le pouvoir de juxtaposer en un seul lieu réel plusieurs espaces, plusieurs emplacements qui sont en eux-mêmes incompatibles » (FOUCAULT, 1994 : 758). Il l'oppose à l'utopie, au non-lieu, qui n'existe que dans l'imaginaire. Ceci s'intègre dans un courant de la pensée du $20^{\text {e }}$ siècle qui introduit, à côté des espaces géographiques « réels », des espaces qu'on pourrait désigner comme «théoriques » ou « imaginaires », espaces qui sont tout de même intensément liés aux espaces réels. C'est aussi en quelque sorte une manière de repenser le modèle historique «fondé sur l'idée d'un progrès linéaire et continu » (COLLOT, 2014 : 16). En effet, «l'Histoire elle-même tend à se spatialiser » constate Michel Collot (COLLOT, 2014 : 16). Mais le changement ne peut être décrit comme un simple déplacement d'une perception du temps vers celle de l'espace, puisque c'est l'espace même qui est perçu différemment. Face à l'espace unifiant de Kant, Bertrand Westphal constate que «l'espace est 
aujourd'hui voué à l'hétérogène. C'est l'opinion de toute la critique postcolonial ou francophoniste, pour qui l'espace est sujet aux tensions contradictoires qui naissent de systèmes de représentation incompatibles »(WESTPHAL, 2007 : 70). On assiste «à la pantopie, à l'espace total» (WESTPHAL, 2007 : 71) qui dans une description de Michel Serres est le lieu de «tous les lieux en chaque lieu et chaque lieu en tous les lieux, centres et circonférences, entretien global » (WESTPHAL, 2007 : 71). L'espace humain de la géocritique est «hétérotopique», c'est-à-dire « hétérogène et combinatoire » (WESTPHAL, 2000 : 24).

Après le linguistic turn (le tournant linguistique) - qui dans les années 1960 proposait une nouvelle approche analytique de la critique littéraire : une approche de fondement linguistique qui consistait notamment à dissocier le texte de son contexte référentiel et de l'étudier par rapport à lui-même et à d'autres textes (analyse intra- et intertextuelle), dans la langue et par la langue - nous assistons à un nouveau changement de perception dans la critique littéraire, déterminé par la géocritique ou la géopoétique et qui se manifeste dans le spatial turn (le tournant spatial). Cette approche tente de réintégrer la référentialité dans l'analyse critique des textes littéraires en y ajoutant une dimension géographique. Elle le fait à partir des rapports réciproques entre la littérature et la géographie. Dans l'introduction du recueil The Spatial Turn, Interdisciplinary perspectives, Barney Warf et Santa Arias, les éditeurs de ladite publication, expliquent que la réinsertion de l'espace dans les sciences sociales et humaines est fortement liée à des changements économiques et sociaux dans l'histoire. Parmi ces changements qui affectent notre perception du temps et de l'espace, ils mentionnent notamment le capitalisme qui, en accélérant la production matérielle, abolit les anciens espaces géographiques et crée de nouveaux paysages soumis à la domination du temps (l'époque du temps); ceci est suivi de la société d'information d'un capitalisme postmoderne qui permet, par le surplus d'information, l'accès à une multitude d'espaces et de lieux, et qui trouve sa force «dans les liens entre des lieux, leur connexion entre eux (l'époque de l'espace)» («in the linkages among places, their interconnectedness », in WARF-ARIAS, 2009: 4). Selon eux, l'apparition de l'espace dans les sciences humaines est due à son importance inscrite dans la réalité humaine postmoderne. En reprenant les idées d'Henri Lefebvre, les auteurs de l'Introduction soulignent que «l'espace doit être pensé non seulement en tant qu'objet matériel et concret, mais aussi comme un objet idéologique, vécu et subjectif» (« space must be understood not simply as a concrete, material object, but also as an ideological, lived, and subjective one », in : WARF-ARIAS, 2009 : 3). L'espace est une construction sociale, et en tant que telle est apte à représenter la réalité humaine et à influencer le regard de notre époque sur les réalités socioculturelles. En raison de la hausse d'intérêt pour l'espace sous toutes ses formes, la géographie en tant que jeune science, qui jusque-là avait été bénéficiaire des apports des autres sciences, est devenue une de leurs sources qu'il s'agisse de la littérature, des sciences culturelles, de la sociologie, des sciences politiques, de l'anthropologie, de l'histoire ou de l'histoire de l'art.

Pour envisager une lecture spatiale de la littérature nous recourrons au concept opératoire de paysage de Michel Collot et à celui de carte dans la géophilosophie de Gilles Deleuze et Félix Guattari. Tout en restant sur le chemin tracé par la géocritique et les nouvelles voies esquissées par le tournant spatial, nous espérons 
donner un cadre théorique dans lequel l'analyse d'un texte littéraire s'avère fructueuse.

Pour comprendre en quoi le paysage et la carte sortent des simples cadres de la géographie, il faut prendre en compte le contexte philosophique dans lequel ils acquièrent un sens élargi et s'interprètent en tant que figures de la pensée. Herman Parret, dans son article Spatialiser haptiquement : de Deleuze à Riegl, et de Riegl à Herder, parle des différentes perceptions de l'espace. Il souligne que «la spatialisation est organisée à partir du corps, de ses vécus et de ses esthésies » (PARRET, 2009). Il ajoute que «la spatialisation ne peut être qu'affective, que tout espace comme lieu d'identité d'un sujet, est un espace signitif, symbolique, onirique, hallucinatoire, angoissant, intime ou étranger, un espace radicalement sémiotisé, certainement pas l'espace naturalisé de la physique et de la géométrie » (PARRET, 2009).

À première vue, deux perceptions s'opposent : l'œil objectif du cartographe à celui de l'habitant; la planification urbaine de la ville à l'expérience personnelle. «Le pilote ne voit qu'une carte [au lieu d'un paysage] » (RADNÓTI 1975 : 101) comme résume Miklós Radnóti, poète hongrois, cette opposition dans un de ses poèmes les plus connus, daté de 1944, Je ne sais... (RADNÓTI 1975 : 101). Nous citons quelques extraits de ce poème pour illustrer cette pensée :

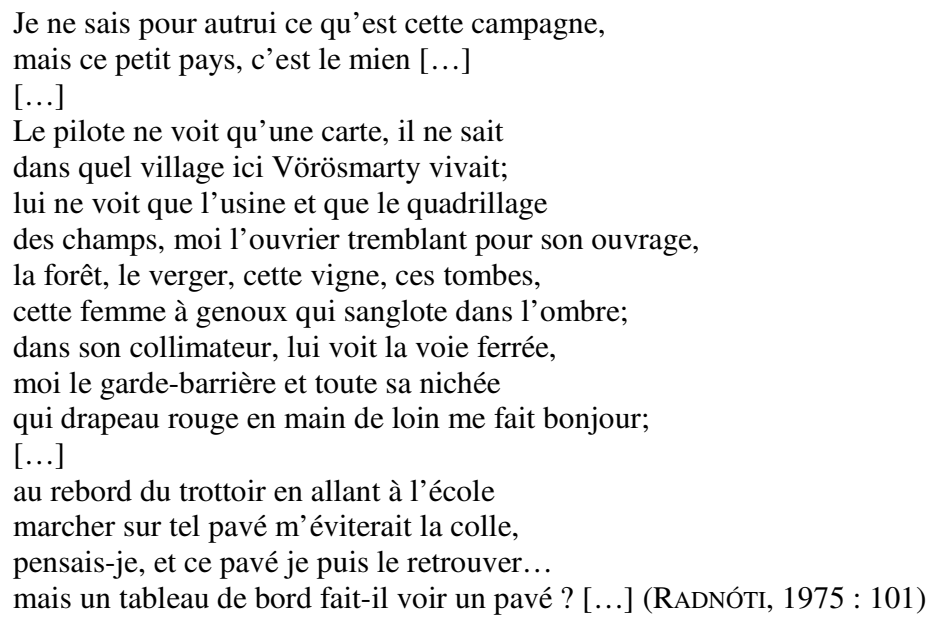

Radnóti oppose, dans son poème, deux perceptions de son pays natal : celle d'autrui, vue d'en haut, du pilote, du cartographe, de l'œil objectif de l'étranger qui ne voit que des frontières, des chemins ferroviaires, des champs, des bâtiments, des usines, bref, des lignes et des points qui tracent la surface d'une carte ; et sa propre vision personnelle du paysage qui l'entoure, avec ses habitants, ses histoires, ses souvenirs, sa mémoire, ses odeurs, ses couleurs et ses sensations. L'une est la vue de loin, distanciée optiquement; tandis que l'autre est celle du proche, du trop proche parfois, haptique. Un des exemples mémorables tiré du poème, celui du pavé au bord de la route, met en contexte cette différence : «au rebord du trottoir en allant à l'école / marcher sur tel pavé m'éviterait la colle, / pensais-je, et ce pavé je puis le retrouver... / Mais un tableau de bord fait-il voir un pavé ?» 
(RADNÓTI, 1975 : 101) Ce pavé, que l'on ne peut voir d'en haut, contient un ensemble de souvenirs d'enfance et de significations, une fois vu de près. Comme si ce petit objet renfermait en lui tout un univers, et en tant que chronotope bakhtinien une temporalité et espace autre que la temporalité linéaire de la pensée rationnelle. Et même, peut-être, ce pavé aura-t-il le même effet que la fameuse madeleine de Proust, si le poète marche dessus.

Dans ce petit extrait, nous pouvons remarquer l'opposition thématique des deux types de visions sur laquelle se base la structure du poème. La narration oscille entre ces deux perceptions : entre la carte et le paysage. Mais aussi entre deux modèles d'espaces : entre l'espace optique et l'espace haptique, ou autrement entre le strié et le lisse.

Dans Pour une géographie littéraire, (COLLOT, 2004) Collot part du concept de paysage pour ensuite élaborer une approche plus synthétique, susceptible d'intégrer la méthodologie des approches géographiques de la littérature et de la géocritique qu'il appelle la géographie littéraire. Cependant, il reste ancré dans sa penséepaysage sans pour autant donner une méthodologie qui pourrait nous servir dans une analyse approfondie des textes. Nous reprenons toutefois son concept de paysage qui semble donner un cadre suffisamment utile pouvant servir à une lecture géocritique de la littérature. Si le paysage se révèle être l'un des éléments fondamentaux de sa pensée, c'est qu'il résume toute une philosophie de l'art et de la création artistique qui lui est propre. Puisque la "poéticité du paysage [...] déborde les frontières entre les genres »(COLLOT, 2005 : 16), la notion de paysage se répand à travers toute création artistique ou littéraire. Le paysage ne se limite pas à la simple représentation picturale, même s'il trouve ses origines dans la peinture, mais il apparaît dans d'autres domaines artistiques également, dont la littérature et l'écriture seront un médium important. Il faut comprendre que le paysage n'est pas simplement une représentation du pays qui se donne à nos yeux, mais qu'en effet, le paysage devient un trope de la pensée. Ce n'est pas une simple représentation de ce qui nous entoure mais une compréhension du monde, à travers le point de vue du sujet qui le conçoit ; le monde qui comprend en même temps le sujet lui-même, puisque le monde ne se trouve pas en face de celui qui l'observe, mais l'entoure et le contient; et le sujet qui fait partie de ce monde même qu'il regarde. C'est l'espace vécu, intime du sujet qui se forme par le rapport sensible entre lui et le monde.

L'intimité de l'espace que Collot souligne dans son étude des méthodes d'analyses littéraires géographiques est donc reflétée dans le concept de paysage. L'importance qu'il accorde au sujet percevant est déjà apparente dans un de ses premiers articles, Point de vue sur la perception des paysages, dans lequel il examine cette notion des points de vue psycho-physiologique, phénoménologique et psychanalytique (COLLOT, 1986). C'est par rapport aux différentes notions de l'espace qu'il cherche à situer le paysage :

On ne peut parler du paysage qu'à partir de sa perception. En effet, à la différence d'autres entités spatiales, construites par l'intermédiaire d'un système symbolique, scientifique (la carte) ou socio-culturel (le territoire), le paysage se définit d'abord comme espace perçu : il constitue «l'aspect visible, perceptible de l'espace » (COLLOT, 1986: 211) 
Il semble donc que ce serait le caractère subjectif du paysage qui le différencierait des «autres entités spatiales »; il n'empêche toutefois qu'il relève autant de l'objectif que du subjectif :

Mais si cette perception [du paysage] se distingue des constructions et symbolisations élaborées à partir d'elle, et réclame d'autres méthodes d'analyse, son apparente immédiateté ne doit pas faire oublier qu'elle ne se borne pas à recevoir passivement les données sensorielles, mais les organise pour leur donner un sens. Le paysage perçu est donc déjà construit et symbolique. (COLLOT, $1986: 211$ )

Il s'agit de voir le paysage comme une construction subjective qui, néanmoins, se crée par l'intermédiaire de la langue et de la pensée symbolique. Le paysage est toujours médiatisé, par la langue ou par d'autres pratiques artistiques, c'est pourquoi sa construction garde un certain formalisme pour devenir médium de la pensée. Toutefois, on ne peut pas dire qu'il soit subjectif ou objectif, le penser en termes de dichotomie le limiterait et bornerait la compréhension de cette notion. Cette distinction cartésienne présume un sujet qui se trouve en face de l'objet observé, un esprit qui est fondamentalement séparé de son corps physique, qui se trouve dans le monde, lui aussi à son tour, physique. Or, la conscience phénoménologique, au contraire, ne peut se différencier du monde qu'elle conçoit, puisqu'elle comprend déjà en soi la caractéristique d'être-au-monde (Voir l'ontologie phénoménologique de Martin Heidegger dans l'Être et temps). C'est pourquoi, le paysage, dans la conception de Collot, ne peut être réduit à une simple représentation du monde, puisqu'il s'agit d'une compréhension du monde d'un sujet, qui se trouve déjà dans ce monde, et qui se comprend à travers ce monde même qu'il décrit. Il y a un rapport de réciprocité entre le monde et l'œuvre. Le paysage s'identifie avec l'écriture, et même avec l'écrivain. L'observateur se dissout dans l'observé, dans le trajet parcouru, le paysage se confond avec celui qui le perçoit :

la phénoménologie montre [...] que cette solidarité entre paysage perçu et sujet percevant joue à double sens : en tant qu'horizon, le paysage se confond avec le champ visuel regardant, mais en retour, toute conscience étant conscience de... le sujet se confond avec son horizon et se définit comme être-au-monde (COLLOT, 1986 : 212).

Effectivement, l'écriture arrache le moi à son moi qui, en conséquence, se perd dans le paysage. C'est pourquoi la lecture d'œuvres littéraires, ainsi que toute réception et contemplation d'œuvres d'art nous fait découvrir, selon la théorie des mondes possibles, des univers différents. Et à travers ces univers possibles, et en dépliant les signes de l'art, autrui, qui se trouve à l'autre bout de cette représentation artistique, se manifeste également. C'est ainsi qu'on peut éviter le piège du solipsisme artistique.

Franco Moretti a étudié, dans plusieurs de ses œuvres, la géographie des littératures. Or, Moretti s'en tient à l'étude pure et simple des références géographiques des livres sans pour autant interroger comment les textes littéraires font usage de la géographie. Il justifie cette approche par des raisons philologiques en soulignant la dimension référentielle des textes. Cette démarche s'inscrit plutôt dans une géopolitique de la littérature que dans une géocritique ou géopoétique ; 
géopolitique qui s'apprête à faire l'étude des centres et des périphéries, et celle des rapports concrets entre la géographie et la création littéraire. Dans l'introduction de son Atlas du roman européen (MORETTI, 2000), il justifie sa démarche en affirmant qu'il faut aller au-delà d'une simple représentation de la littérature dans des espaces géographiques, et arrive à la conclusion qu'en analysant l'aspect géographique des textes et des livres, on dévoile un aspect caché du champ littéraire. Moretti ne s'écarte pas de l'abstraction. Dans son dernier ouvrage, Graphes, cartes et arbres (MORETTI, 2008), il fait l'analyse de phénomènes littéraires à partir des diverses formes abstraites. Il admet, dans cet essai, qu'il a toujours eu tendance à l'abstraction et que la critique n'a pas eu tort de le lui reprocher que, parfois, il usait plutôt d'une géométrie que d'une géographie. Cependant, il ne trouve pas cela problématique, puisque l'espace, comme il l'explique, a tendance à se scinder en géométrie et en géographie, et à osciller entre ces deux pôles. Son objectif est, encore une fois, de relever une dimension cachée devant l'étude des œuvres en soi en mariant l'histoire littéraire avec les formations géographiques et géométriques, qu'il résume déjà dans les premières lignes de l'introduction :

Le titre de ce petit livre mérite quelques mots d'explication. Pour commencer, il s'agit d'un essai d'histoire littéraire: il s'attache à la littérature, ce vieux territoire depuis longtemps abandonné par le new historicism et les cultural studies. Mais à l'intérieur de ce vieux territoire, un nouvel objet d'étude : à la place des œuvres singulières, concrètes, un trio de constructions artificielles - graphes, cartes et arbres - dans lesquelles la réalité du texte subit un processus de réduction et d'abstraction délibéré. «Lecture de loin »(distant reading), ainsi ai-je nommé ce type d'approche il y a quelques années - où la distance n'est pas un obstacle, mais une forme spécifique de connaissance : un nombre plus réduit d'éléments, d'où un sens plus aigu de leur interconnexion globale. Organisations, relations, structures. Formes. Modèles (MORETTI, 2008 : $33)$.

La question se pose de savoir si un texte (et non pas un livre comme entité physique) détient réellement un rapport avec la réalité, ou bien si cette référentialité reste à un certain point fictionnel ou imaginaire. Fictionnel ou imaginaire dans le sens où un vrai rapport ne peut jamais être établi entre le réel et l'imaginaire, mais que seules les représentations des phénomènes du réel peuvent être présentes dans l'imaginaire. Une approche purement géographique et référentielle ne peut en effet être novatrice puisqu'elle reste ancrée dans la dualité de géographie et littérature. Pour pouvoir analyser les textes littéraires, et tenir compte de leur littérarité, la géographie doit se rendre critique, et doit emprunter à la méthodologie de la critique littéraire. Comme Brosseau l'a bien remarqué dans Des romans-géographe, on doit établir un dialogue entre les deux disciplines. C'est le problème de la carte géographique, qui, selon Collot, ne peut représenter ou illustrer l'«imaginaire cartographique » qui «au lieu de réduire l'espace littéraire à ses coordonnées géographiques, ouvre en lui des horizons indéfinis » (COLLOT, 2014 : 84). Selon lui, la carte est une représentation factuelle et objective des données extraites du texte littéraire, alors qu' «on ne peut trouver ni figurer [le paysage] sur aucune carte » (COLLOT, $2014: 85)$; le paysage se déploie à travers une lecture proche et profonde. Or, la carte ne doit pas être écartée de l'analyse littéraire, il faudra au contraire intégrer les paysages dans une cartographie de l'imaginaire. 
Collot condamne la carte face au paysage pour sa prétendue objectivité et son manque de profondeur puisque, selon lui, elle est essentiellement bidimensionnelle. Il présume que «l'espace de la carte n'est construit à partir d'aucun point de vue privilégié » (COLLOT, 1986: 212), autant qu' " [i]l ignore la perspective horizontale, si bien que tous les objets s'y trouvent reproduits à la même échelle » (COLLOT, 1986 : 212). L'espace de la carte est essentiellement lié au regard optique, opposé à l'haptique, «[i]l est vu du dehors et d'en haut, [...] il est réduit à deux dimensions » (COLlOT, 1986: 212). En conclusion, il affirme que «[1]'espace du paysage, organisé à partir d'un point de vue unique et selon une perspective horizontale, s'oppose en tous points à celui de la carte » (COLLOT, 1986 : 212). Or, Collot oublie nécessairement que la carte non plus, en tant que produit de la cartographie - une certaine écriture de l'espace - ne peut entièrement se dissocier de son créateur, et ne peut ainsi être purement objective. Dans cette approche du paysage et de la carte, nous ne sommes pas d'accord avec le point de vue de Collot, puisque, comme nous espérons le présenter dans ce qui suit, ces deux concepts ne s'opposent pas l'un à l'autre, mais plutôt se trouvent dans un rapport de complémentarité et, effectivement, pour pouvoir donner une analyse approfondie des textes littéraires, il nous faut recourir à ces deux approches, ou plutôt à une approche qui réconciliera les deux concepts.

Nous avons déjà brièvement parlé de la problématique de la carte et de la cartographie, nous abordons à présent ce sujet dans le détail, notamment la révolution apportée dans l'interprétation des cartes et l'apport d'une réévaluation de la cartographie autant au sens concret, qu'à un sens plus élargi, métaphorique. La carte, si on la considère dans un sens métaphorique, peut en effet être pluridimensionnelle, tandis que la carte au sens traditionnel est à tort pensée comme bidimensionnelle à cause du médium (le papier) qui détermine sa perception. De fait, un changement est à l'œuvre dans la perception de la carte, car on peut l'interpréter comme un phénomène multidisciplinaire et non comme un simple dessin représentant deux dimensions de l'espace dans la géographie. Elle ne peut en effet être purement objective vu qu'elle comprend en tout temps des éléments du contexte culturel dans lequel elle a été créée ainsi que le point de vue de celui ou ceux qui l'ont créée. La carte réintroduit une certaine approche structuraliste dans une analyse purement thématique de la spatialité littéraire. Elle ne s'oppose plus simplement au paysage, elle réalise au contraire une relation de complémentarité. Écrire c'est cartographier aussi en même temps. L'écriture à travers laquelle se déploient les paysages littéraires peut être conçue comme un certain cadre, tout comme la carte l'est pour l'espace. On le sait : ce qu'on appelle «tournant spatial » a certainement eu effet sur la géographie également, ainsi que sur la perception de la cartographie puisque les actes de cartographie sont « des actes de visualisation, de conceptualisation, d'enregistrement, de représentation et de création graphique d'espaces» (" acts of visualizing, conceptualizing, recording, representing and creating spaces graphically », in : COSGROVE, 1999 : 1). De plus, la cartographie s'inscrit dans les modes de communication interpersonnels, étant donné que

« [c]artographier c'est, d'une manière ou d'une autre, prendre les mesures d'un monde, et ce n'est pas simplement les prendre, mais représenter ces mesures ainsi prises d'une telle manière 
qu'elles puissent être communiquées entre différentes personnes, temps et lieux » (« To map is in one way or another to take measure of a world, and more than merely take it, to figure the measure so taken in such a way that it may be communicated between people, places or times ", in COSGROVE, 1999 : 1-2).

Quant à la théorie des mondes possibles, elle semble s'inscrire dans cette perspective en ce qu'elle souligne la potentialité imaginaire infinie de créer des possibilités de monde. L'actualisation d'un phénomène (une carte, par exemple) ne signifie pas l'exclusion du potentiel qui l'habite. Le possible fait aussi partie de l'actuel, même si ce n'est pas son côté visible, sensible. C'est en effet ce que l'écriture accentue. L'écriture, tout comme la carte, est un tracé, dans le temps et dans l'espace, de la pensée. C'est la mouvance pluridimensionnelle qui définit la carte, le fait qu'elle n'est pas assignable à un point fixe. Elle ne peut le faire.

En effet, le texte et la carte montrent plusieurs similarités en ce qui concerne leur structure. Robert Tally dans son essai, Spatiality, décrit un système de spatialisation de la littérature qui comprend la cartographie littéraire et la géographie littéraire comme deux aspects de ce phénomène. Il décrit le travail de l'écrivain comme une démarche de cartographie; quant au lecteur, il fait une géographie littéraire de l'œuvre qu'il lit :

Si les écrivains cartographient les espaces réels et imaginaires de leur monde à travers de multiples moyens littéraires, il s'ensuit que les lecteurs font également partie de ce projet cartographique au sens large. Un lecteur de carte est aussi un lecteur de texte après tout, et le lecteur d'une carte littéraire conçoit l'espace, trace la trajectoire, et se repère dans ce monde représenté qui lui est adressé sur cette carte. De plus, un lecteur n'est jamais un simple récipient passif des messages spatiaux transmis par la carte ou le texte, mais influence activement les sens éphémères et changeants de la carte. Ainsi, à la cartographie littéraire de l'écrivain s'ajoute la géographie littéraire du lecteur. Le lecteur critique devient une sorte de géographe qui interprète activement la carte littéraire pour y déployer de nouvelles cartes, dès lors invisibles.

(If writers map the real and imagined spaces of their world in various ways through literary means, then it follows that readers are also engaged in this broader mapping project. A map-reader is also a reader of a text, after all, and the reader of a literary map also envisions a space, plots a trajectory, and becomes orientated to and within the world depicted there. What is more, the reader is never simply a passive receptacle for the spatial messages transmitted by the map or text, but actively determines the often shifting and transient meanings to be found in the map. To the writer's literary cartography, we might add the reader's literary geography. The critical reader becomes a kind of geographer who actively interprets the literary map in such a way to present new, sometimes hitherto unforeseen mappings. In : TALLY, $2013: 79)$

Cette observation inclut dans son corpus tout texte et non seulement les genres aux traits géographiques apparents. Sans doute, certains genres littéraires semblent être plus «spatiaux » que d'autres, il n'empêche que toute écriture comporte une certaine spatialité, soit-elle liée à des espaces réels ou fictifs.

La carte n'est pas en soi entièrement objective, puisqu'elle détient, comme toute autre écriture, la possibilité d'un sujet écrivant ou lisant. Une carte dont l'objectivité serait incontestable (probablement une carte à grande échelle, au 1/1 même) serait des plus inutiles au monde, comme la carte des cartographes citée par Jorge Luis Borges dans De la rigueur dans la science, texte que Borges attribue à un écrivain du $17^{\mathrm{e}}$ siècle, et que nous citons dans son intégralité : 
En cet empire, l'Art de la Cartographie fut poussé à une telle Perfection que la Carte d'une seule Province occupait toute une Ville et la Carte de l'Empire toute une Province. Avec le temps, ces Cartes Démesurées cessèrent de donner satisfaction et les Collèges de Cartographes levèrent une Carte de l'Empire, qui avait le Format de l'Empire et qui coöncidait avec lui, point par point. Moins passionnées pour l'Étude de la Cartographie, les Générations Suivantes réfléchirent que cette Carte Dilatée était inutile et, non sans impiété, elles l'abandonnèrent à l'Inclémence du Soleil et des Hivers. Dans les Déserts de l'Ouest, subsistent des Ruines très abîmées de la Carte. Des Animaux et des Mendiants les habitent. Dans tout le Pays, il n'y a plus d'autre trace des Disciplines Géographiques. (Suarez Miranda. Viajes de Varones Prudentes, Lib. IV, Cap. XIV, Lérida 1658.) (BORGES, 1994 : 107).

Ou même cette carte non dépliée de Lewis Carroll dans le chapitre XI de Sylvie et Bruno (suite et fin) :

- Voilà une chose que nous avons apprise de votre pays, dit Mein Herr, faire des cartes. Mais nous l'avons poussée beaucoup plus loin que vous. À votre avis, quelle serait la plus grande échelle de carte utile?

- Je dirais un cent millième, un centimètre au kilomètre.

- Seulement un centimètre ! s'exclama Mein Herr. Nous avons atteint cela très vite. Puis nous avons tenté dix mètres au kilomètre. Puis vint l'idée grandiose! Nous avons réellement fabriqué une carte du pays, à l'échelle d'un kilomètre au kilomètre !

- Vous en êtes-vous beaucoup servi ? demandai-je.

- Elle n'a jamais encore été déroulée, dit Mein Herr ; les fermiers ont fait des objections ; ils ont dit que ça couvrirait tout le pays et que ça cacherait le soleil ! Aussi nous utilisons le pays luimême comme sa propre carte, et je vous assure que ça marche aussi bien. (CARROLL, $1990: 692$ )

Par ailleurs, comme tout système symbolique, la carte non plus ne peut être sans un lecteur qui la perçoit en tant que carte, ou dans le cas de l'écriture comme texte. On l'a vu avec le structuralisme, le texte est sujet à un champ d'interprétations infinies. Dans une approche sémiotique (qui déplie le sens, la multitude de sens du signe de la représentation, la multitude d'interprétations inhérentes, présentes dans le signe), la carte occupe le même rôle que l'écriture. Ce point de vue s'inscrit également dans la théorie des mondes possibles qui accentue l'importance équivoque des mondes possibles à côté du monde actuel, comme autant de mondes potentiels qui se déploient dans la représentation du monde qui nous entoure.

Pour décrire l'intimité de l'espace et de la perception, Deleuze introduit le terme de « haptique » en parallèle de l'optique. Il opte pour ce néologisme plutôt que pour l'adjectif « tactile » puisqu'il trouve que, dans ce dernier, il y a une prédominance, même une exclusivité de la perception du monde à travers le toucher. Or, l'haptique peut être à la fois visuel, auditif et tactile. L'espace optique c'est l'espace strié, c'est l'espace de la représentation, de l'organisé, de la distance; tandis que l'espace haptique c'est l'espace lisse, l'espace du vécu, du proche.

Gilles Deleuze et Félix Guattari consacrent un chapitre entier à l'espace strié et à l'espace lisse dans Mille Plateaux. En décrivant le modèle musical, ils disent :

le strié, c'est ce qui entrecroise des fixes et des variables, ce qui ordonne et fait succéder des formes distinctes, ce qui organise les lignes mélodiques horizontales et les plans harmoniques verticaux. Le lisse, c'est la variation continue, c'est le développement continu de la forme, c'est la fusion de l'harmonie et de la mélodie au profit d'un dégagement de valeurs proprement rythmiques, le pur tracé d'une diagonale à travers la verticale et l'horizontale. (DeLEuzE GUATTARI, $1980: 597)$ 
Ils ajoutent en parlant du modèle maritime que

dans l'espace strié comme dans l'espace lisse, il y a des points, des lignes et des surfaces [...]. Or dans l'espace strié, les lignes, les trajets, ont tendance à être subordonnées aux points : on va d'un point à un autre. Dans le lisse, c'est l'inverse : les points sont subordonnés au trajet. (Deleuze - GuatTARI, $1980:$ 597)

Il faut toutefois souligner qu'il ne s'agit pas d'une simple opposition entre ces deux espaces, ni d'une opposition entre espace hétérogène et espace homogène. L'espace lisse est loin d'être homogène, on le voit bien dans la description du modèle technique de tissage, dans les exemples que les deux auteurs donnent pour illustrer ces deux types d'espaces : le strié c'est le tissu, le tricot, la broderie ; tandis que le lisse c'est le feutre, le crochet, le patchwork.

Même si le paysage n'est pas l'équivalent exact de l'espace haptique, il s'avère que le paysage, dans la conception de Collot, s'inscrit dans la géophilosophie deleuzo-guattarienne en tant qu'il participe à la fois de l'espace optique et haptique. La carte, en revanche, dans cette opposition, ne peut être qu'optique, même si, dérivée du paysage, elle garde une part de subjectivité.

Jusqu'ici nous avons présenté un concept de la carte - face au paysage - qui accentue son aspect objectif : le regard objectivant des autres. La carte dans ce sens est la prise de distance par rapport à son objet représenté, l'abstraction de l'objet étudié, l'objectif. Or, la géophilosophie telle que conçue par Deleuze et Guattari dissout l'opposition du sujet et de l'objet dans le mouvement de la déterritorialisation et de la reterritorialisation. Dans leur pensée, la carte se comprend par rapport au calque qui n'est qu'une simple reproduction du réel (pensons à cette fameuse carte au 1/1 !). À la différence de celui-ci, la carte représente un certain point de vue, une représentation interprétée de la réalité, c'està-dire un tracé original du réel. Tout comme un livre le ferait puisqu' "[é]crire n'a rien à voir avec signifier, mais avec arpenter, cartographier, même des contrées à venir » (DELEUZE - GUATTARI, $1980: 11)$.

Chez Deleuze, « [1] a carte exprime l'identité du parcours et du parcouru. Elle se confond avec son objet, quand l'objet lui-même est mouvement. » (DELEUZE, 1993 : 81) Dans ce contexte, la carte n'est donc pas nécessairement l'objectivation des espaces humains, mais une certaine compréhension des espaces qui nous entourent, de l'environnement, une image de la pensée, une interprétation du monde qui nous entoure. C'est dans ce tracé que s'inscrit la démarche de Collot, la géographie littéraire, susceptible de synthétiser les trois approches esquissées dans les premiers chapitres de Pour une géographie littéraire, et qui sert de cadre pour ses analyses critiques de la littérature. L'archipel comme phénomène géographique devient ainsi un des principes organisationnels de la pensée, une certaine structure rhizomatique, qui, à la surface, semble être morcelée, fragmentée, un ensemble d'éléments hétérogènes qui ne communiquent pas entre eux, mais qui, dans leur fondement, forment bel et bien une structure, non pas celle de la hiérarchie, de l'arbre, mais celle du rhizome dont les éléments sont interconnectés par autant de lignes de force et de fuite et forment ainsi une unité essentielle. 


\section{BIBLIOGRAPHIE}

Borges, J. L. (1994), Histoire universelle de l'infamie. : Histoire de l'éternité, Paris, Éditions 10/18.

CARroll, L. (1990), Sylvie et Bruno (suite et fin) in Euvres, Paris, Gallimard.

Collot, M. (1986), «Points de vue sur la perception des paysages » in Espace géographique, t. 15, $\mathrm{n}^{\circ} 3,211-217$.

Collot, M. (2005), Paysage et poésie, Paris, José Corti.

Collot, M. (2014), Pour une géographie littéraire, Paris, Éditions Corti.

Cosgrove, D. (dir.) (1999), Mappings, London (Royaume-Uni), Reaktion Books.

Deleuze, G. - F. GuATtARI (1980), Capitalisme et schizophrénie II, Mille plateaux, Paris, Éditions de Minuit.

Deleuze, G. ; F. GUATTARI (1991), Qu'est-ce que la philosophie ?, Paris, Éditions de Minuit.

Deleuze, G. (1993), Critique et clinique, Paris, Éditions de Minuit.

Foucault, M. (1994), «Des espaces autres » in Dits et écrits IV, Paris, Gallimard, 752-762.

MoretTi, F. (2000), Atlas du roman européen (1800-1900), Paris, du Seuil.

MORETTI, F. (2008), Graphes, cartes et arbres. Modèles abstraits pour une autre histoire de la littérature, Paris, Les Prairies ordinaires.

PARRET, H. (2009), « Spatialiser haptiquement : de Deleuze à Riegl, et de Riegl à Herder $\gg$ in Actes sémiotiques, $\mathrm{n}^{\mathrm{o}} 112$, [En ligne]. http://epublications.unilim.fr/revues/as/2570 (page consultée le 18 mars 2016).

RADNóti M. (1975), Marche forcée : poèmes ; suivis de Le mois des gémeaux, Paris, P.J. Oswald.

TALly, R. T. (2013), Spatiality, New York, Routledge.

WARF, B. ; S. Arias (dir.) (2009), The Spatial Turn, Abingdon (Royaume-Uni), Routledge.

WESTPHAL, B. (2000), La géocritique mode d'emploi, Limoges, PULIM.

WestPhal, B. (2007), La Géocritique : réel, fiction, espace, Paris, Minuit. 